\title{
Electrostatic Interactions between Peptides and the Molecular Chaperone DnaK
}

\author{
W. Liu ${ }^{1}$, D. Bratko ${ }^{1}$, J. M. Prausnitz ${ }^{1,2}$ and H. W. Blanch ${ }^{1}$ \\ ${ }^{1}$ Department of Chemical Engineering, University of California, Berkeley, CA, 94720 \\ ${ }^{2}$ Chemical Sciences Division, Lawrence Berkeley National Laboratory, Berkeley, CA, 94720 \\ January 16, 2003
}

\begin{abstract}
The molecular chaperone DnaK prevents intracellular protein misfolding and aggregation by transiently binding with newly synthesized polypeptides and protein folding intermediates. DnaK preferentially binds to peptides with basic residues (Arg/Lys) present on the outside of a hydrophobic core. The electrostatic contribution toward DnaK/peptide binding was determined by measuring the dissociation constant of DnaK complexes with two fluorescein-labeled peptides (f-NRLLLTG and f-NALLLTG) using fluorescence anisotropy. The measured dissociation constants, $K_{d}$, differ significantly at low ionic strength: at $20 \mathrm{mM}$ phosphate buffer, $K_{d}$ for DnaK and f-NRLLLTG is $0.2 \mu \mathrm{M}$ while that for DnaK and f-NALLLTG is $1 \mu \mathrm{M}$. This difference, attributed to stronger Coulombic binding in the case of f-NRLLLTG, vanishes at high ionic strength due to electrostatic screening. For f-NRAAATG, no interaction with DnaK was apparent, showing that hydrophobic interactions are essential in chaperone/peptide binding. The electrostatic contribution to the interaction between DnaK and NRLLLTG is interpreted
\end{abstract}


in terms of an approximate analytic model for the potential of mean force between DnaK and the peptide. The calculated electrostatic binding free energy, $\Delta G_{\text {ele }}$, of about -1.1 $\mathrm{kcal} / \mathrm{mol}$, is in good agreement with the experiment result for low salt concentration, as obtained from the ionic-strength dependence of the measured dissociation constants. Our analytic model provides a useful estimate of electrostatic effects which can be exploited for control of protein-protein interactions.

Keywords: Molecular chaperone, DnaK, Peptides, Fluorescence anisotropy, Coulombic interactions 


\section{Introduction:}

Molecular chaperones perform several functions including stabilizing newly synthesized polypeptides, translocating nascent protein chains and refolding misfolded proteins (Gething and Sambrook, 1992; Hartl, 1996). DnaK is the Hsp70 (70 $k D a$ heat-shock protein) molecular chaperone of Escherichia coli, containing a substrate-binding domain and an ATPase domain. Substrate binding and release are driven by switching between the low-affinity ATP-bound state and the high-affinity ADP-bound state (Langer et al., 1992).

Interactions contributing to DnaK/substrate binding include hydrophobic forces, hydrogen bonds, van der Waals forces, electrostatic interactions and structural elements (an arch formed by DnaK residues $\mathrm{Met}^{404}$ and $\mathrm{Ala}^{429}$ encloses peptide backbone in DnaK substratebinding pocket) (Zhu et al., 1996; Rüdiger et al., 1997a; Wang et al., 1998; Tardieu et al., 1999; Mayer et al., 2000). A previous study of the substrate specificity of DnaK has shown that the peptide-binding motif consists of a hydrophobic core of four to five residues enriched particularly in Leu, and two flanking regions enriched in basic residues. Acidic residues are excluded from the core and disfavored in the flanking region. In folded proteins, these interaction sites are mostly buried and primarily found in the $\beta$-sheet region (Fourie et al., 1994; Gragerov et al., 1994; Rüdiger et al., 1997b).

Electrostatic forces often play a key role in protein-protein interactions. Clusters of charged and polar residues located on protein surfaces can stabilize the protein-protein complex (Sheinerman et al., 2000). The preference of DnaK to bind positively charged peptides indicates that electrostatic interactions enhance specific DnaK/substrate recognition, even though the binding occurs in the DnaK hydrophobic pocket. Unlike hydrophobic interactions, electrostatic 
attraction is relatively easy to control by varying solution conditions such as $\mathrm{pH}$ and ionic strength. Control of electrostatic interactions therefore provides a powerful tool that can be exploited in the design and optimization of a variety of processes affected by interprotein binding. The goal of the present study is to elucidate the role of electrostatics in forming molecular chaperone/substrate complexes and to develop and test an analytic model that can provide a rapid estimate of the electrostatic binding free energy for almost globular, charged proteins with strong dipolar interactions.

We measured the dissociation constants between DnaK and fluorescein-labeled peptides, (f-NRLLLTG and f-NALLLTG), at several ionic strengths using fluorescence anisotropy. The dissociation constant of ionized peptide f-NRLLLTG and DnaK showed a strong dependence on ionic strength. The binding strength of a nonionic peptide, f-NALLLTG, and DnaK showed no significant dependence on salt concentration and was similar to that for f-NRLLLTG and DnaK under screened electrostatic conditions. We obtain the electrostatic contribution to the free energy of DnaK/peptide binding from the difference between measured dissociation constants for DnaK and the two peptides at low and high ionic strengths. Experimental results are used to test an analytic model for the electrostatic potential of mean force between DnaK and peptide NRLLLTG. The measured electrostatic free energy and the calculated contact value of the potential of mean force are in a good agreement, providing consistent estimates of the relative contributions from electrostatic and non-electrostatic interactions in DnaK/peptide binding.

A variety of different methods are available to calculate the electrostatic free energy between protein molecules (Phillies, 1974; Gilson et al., 1988; Nicholls and Honig, 1991; Nicholls et al., 1991; Coen et al., 1995; Coen et al., 1996; McClurg and Zukoski, 1998; Sader and Lenhoff, 1998; Asthagiri et al., 1999; Farnum and Zukoski, 1999; Neal et al., 1999; Grant, 
2001; Bratko et al., 2002). Typically, these methods rely on a dielectric continuum representation for the solvent (Friedman, 1985). The description of the interacting solutes, on the other hand, varies from the highly simplified hard-sphere approximation to nearly atomistic descriptions of the protein; an example of the latter is provided by the highly sophisticated DelPhi (Nicholls and Honig, 1991) and GRASP (Nicholls et al., 1991) computation packages. Application of these models is, however, limited by computational requirements and the need for precise structural input for all interacting components, including information concerning conformational changes associated with the binding process. In the absence of complete and accurate input information, free-energy calculations often require arbitrary scaling procedures (Novotny et al., 1997; Kasper et al., 2000) that limit the validity of computed results to relative comparisons, but do not always suffice for determinations of absolute thermodynamic quantities of interest.

In the present work, we use a simplified model that represents protein and peptide molecules as roughly spherical particles with charge and dipole moments (Bratko et al., 2002). The long-range protein/protein interaction is described by a DLVO-like theory, with screened charge and dipole interactions described by known analytic expressions based on the linearized Poisson-Boltzmann approximation (Phillies, 1974). The electrostatic potential of mean force between globular proteins is estimated as a sum of charge/charge and distinct angle-averaged charge/dipole and dipole/dipole interactions (Phillies, 1974; Coen et al., 1995), augmented by a generalized expression for the coupling correction (Bratko et al., 2002) which accounts for the fact that orientation probabilities depend on all dipole-related interactions. Good agreement between the model prediction and the measured electrostatic binding free energy between DnaK and peptide NRLLLTG illustrates applicability of the proposed analytic approximation to estimate quantitatively electrostatic binding in protein solutions. 


\section{Materials and Methods:}

\section{DnaK Production and Purification}

The bacteria strain E.coli Top10 harboring plasmid pDnaK (a gift from Professor François Baneyx, the University of Washington) was employed for DnaK production. The cell culture and protein purification processes were performed according to procedures described elsewhere (Cegielska and Georgopoulos, 1989; Kamath-Loeb et al., 1995).

Bacteria were grown overnight at $30^{\circ} \mathrm{C}$ in $\mathrm{LB}$ broth $(10 \mathrm{~g} / \mathrm{L}$ tryptone, $5 \mathrm{~g} / \mathrm{L}$ yeast extract and $5 \mathrm{~g} / \mathrm{L} \mathrm{NaCl}$ ), then incubated in a BiofloШ fermentor (New Brunswick Scientific, Edison, NJ) containing 1.5 L supplemented LB medium ( $0.2 \%$ glucose and $34 \mu \mathrm{g} / \mathrm{ml}$ chloramphenicol). The cells were grown at $30^{\circ} \mathrm{C}$ to the mid-exponential phase (absorbance at $600 \mathrm{~nm}, \mathrm{~A}_{600}, \approx 0.4$ ), induced with $1 \mathrm{mM}$ IPTG, and were harvested at the late-exponential phase. The cell cultures were centrifuged at $8000 \times g$ for $15 \mathrm{~min}$ at $0^{\circ} \mathrm{C}$ and the pellets were stored at $-80^{\circ} \mathrm{C}$.

The frozen cell pellet (about $5 \mathrm{~g}$ wet weight) was resuspended in $20 \mathrm{ml} \mathrm{10 \%}$ sucrose in $50 \mathrm{mM}$ Tris-HCL (pH 8.0), and disrupted using a French press at 10,000 psi. Soluble fractions were immediately clarified by centrifugation at $25,000 \times g$ for $30 \mathrm{~min}$ at $0^{\circ} \mathrm{C}$. The supernatant was precipitated slowly by addition of saturated ammonium sulfate solution to $65 \%$ saturation. After slow stirring for $15 \mathrm{~min}$ at $0^{\circ} \mathrm{C}$, the mixture was centrifuged at $45,000 \times g$ for $30 \mathrm{~min}$ at $0^{\circ} \mathrm{C}$. The protein pellet was slowly dissolved in buffer B (100 mM potassium phosphate, 100 $\mathrm{mM}$ ammonium sulfate, $5 \mathrm{mM}$ mercaptoethanol, $5 \mathrm{mM}$ EDTA, and $10 \%$ glycerol $(\mathrm{v} / \mathrm{v})$ at $\mathrm{pH}$ 7.0) and dialyzed against buffer B overnight. 
The dialysate was chromatographed over a DE52-cellulose anion-exchange column $(3 \times 10 \mathrm{~cm})$ (Whatman, Clifton, $\mathrm{NJ}$ ) at a flow rate of $0.5 \mathrm{ml} / \mathrm{min}$. After loading the protein sample, the column was washed with 2 column volumes of buffer B followed by buffer B-containing 500 $\mathrm{mM} \mathrm{NaCl}$. The relevant fraction was collected, dialyzed against buffer A (25 mM HEPES, $1 \mathrm{mM}$ EDTA, $10 \mathrm{mM}$ 2-mercaptoethanol, $10 \mathrm{mM} \mathrm{NaCl}$ and $3 \mathrm{mM} \mathrm{MgCl}_{2}, \mathrm{pH} \mathrm{8.0)}$ and applied to an ATP-agarose column $(1 \times 7 \mathrm{~cm})$ (Sigma, St. Louis, MO) at a flow rate of $0.5 \mathrm{ml} / \mathrm{min}$. The column was washed with 10 volumes of buffer A and then with 10 volumes of buffer A-containing 500 $\mathrm{mM} \mathrm{NaCl}$. After equilibration of the column with 5 volumes of buffer A, DnaK protein was eluted with 10 column volumes of buffer A-containing $10 \mathrm{mM} \mathrm{Mg-ATP}(\mathrm{pH}$ 7.6). The protein sample was dialysed against TEN500 (20 mM Tris, $1 \mathrm{mM}$ EDTA and $500 \mathrm{mM} \mathrm{NaCl}$ at $\mathrm{pH}$ 7.0) for 3 days with 3 buffer changes to remove free ATP.

The dialyzed sample was passed over a $1 \mathrm{ml}$ Resource Q column (Pharmacia, Piscataway, NJ) followed by a TEN0 to TEN500 salt-gradient elution over $20 \mathrm{ml}$ at a flow rate of $0.5 \mathrm{ml} / \mathrm{min} .10 \mu \mathrm{l}$ aliquots of each collect were resolved on a 4-12\% SDS-PAGE to determine the location of DnaK.

The resulting protein sample was dialyzed against TEN100 buffer for 2 days. The ratio of UV absorbance at wavelength $280 \mathrm{~nm}$ and $260 \mathrm{~nm}\left(\mathrm{~A}_{280} / \mathrm{A}_{260}\right)$ was determined daily to monitor the removal of ATP (if $\mathrm{A}_{280} / \mathrm{A}_{260}>1.5$, the solution was considered to be ATP free (Chaykin, 1966)). Protein concentration was determined by BCA (bicinchoninic acid) (Smith et al., 1985) protein assay (Pierce, Rockford, IL). Protein samples were stored at $-80^{\circ} \mathrm{C}$. 


\section{Peptide Synthesis and labeling}

Three peptides (f-NRLLLTG, f-NALLLTG and f-NRAAATG) were synthesized and fluorescein-labeled at $\mathrm{N}$ terminals by Dr. Michael Moore at the Cancer Research Center, University of California, Berkeley. Electrospray ionization mass spectrometry yielded peptide molecular weights consistent with those based on primary structures.

\section{Fluorescence Anisotropy}

Fluorescence anisotropy provides a method for measuring protein-protein interactions by observing the perturbations in the rotational molecular motion of fluorescent molecules. Due to Brownian motion, the molecular rotational diffusion coefficient $D_{r}$ is (Einstein, 1906; Weber, 1953):

$D_{r}=\frac{R T}{6 \eta V}$

where $R$ is the gas constant, $T$ the absolute temperature, $\eta$ the solution viscosity and $V$ the molecular volume. Upon association with other molecular species, the rotational motion of the fluorescent molecule will be changed because of the increase in its size and can be related to fluorescence anisotropy, $A$, according to Perrin's equation (Lakowicz, 1983):

$$
A=\frac{A_{o}}{1+(R T \tau / \eta V)}
$$

where $A_{o}$ is the limiting anisotropy representing the anisotropy in the absence of rotational molecular motion and $\tau$ is the fluorescence life time of the fluorophore.

Fluorescence anisotropy is measured by first exciting a molecule with vertically polarized light, then observing the fluorescence intensity of emitted light parallel to or perpendicular to the 
direction of polarization of the incident light. The anisotropy is related to these intensities by (Lakowicz, 1983):

$A=\frac{I_{/ /}-I_{\perp}}{I_{/ /}+2 I_{\perp}}$

where $I$ is the emitted light intensity measured along a direction parallel $(\|)$ or perpendicular $(\perp)$ relative to the direction of excitation.

Consider binding interaction between two molecular species, $P$ and $L$ :

$P+L \stackrel{\rightleftarrows}{\longleftarrow} P L$

$P$ in this case represents molecular chaperone DnaK, and $L$ denotes the fluorescent-labeled peptide. At equilibrium, the dissociation constant $K_{d}$ can be expressed as:

$K_{d}=\frac{[P][L]}{[P L]}$

where $[P],[L]$ and $[P L]$ are the concentrations of DnaK, peptide and DnaK-peptide complex, respectively.

When the concentration of DnaK is much higher than that of peptide in solution, the concentration $[P]$ can be approximated by the total DnaK concentration $\left[P_{T}\right]$. The anisotropy is related to the dissociation constant by

$$
A=A_{f}+\left(A_{b}-A_{f}\right)\left[\frac{\left[P_{T}\right]}{K_{d}+\left[P_{T}\right]}\right]
$$

where $A$ is the measured anisotropy in a mixture of free and bound fluorescent molecules with anisotropies $A_{f}$ and $A_{b}$, respectively (Lundblad et al., 1996).

The fluorescent-labeled peptides tumble at rates related to their molecular weight and volume, and display modest anisotropy. However, when the peptides bind to DnaK, their molecular rotation approaches that of DnaK, showing high anisotropy. During fluorescence- 
anisotropy measurement, the fluorescent-labeled peptide concentrations were kept constant while the anisotropy was recorded as a function of increasing DnaK concentration.

\section{Fluorescence-anisotropy Measurement}

Fluorescence-anisotropy measurements were performed using a BEACON 2000 (PanVera, Madison, WI) fluorescence-polarization system. The excitation wavelength was 495 $\mathrm{nm}$ and the emission wavelength was $520 \mathrm{~nm}$. Solutions of DnaK and peptides were incubated at $25^{\circ} \mathrm{C}$ for 2 hours to reach equilibrium. Fig. 1 shows the anisotropy change associated with the binding of peptide f-NRLLLTG and f-NALLLTG with increasing concentrations of DnaK. The fluorescence intensity of peptide solutions was also recorded as a function of DnaK concentration and found to be constant (data not shown) during all measurements, suggesting that fluorescein does not bind with DnaK. The anisotropy data were fitted to a first-order binding equation (Lundblad et al., 1996) using a regression algorithm from Sigma Plot to obtain dissociation constants.

\section{Electrostatic free energy of binding}

To improve our ability to control interprotein interactions, it is useful to develop a model for approximate prediction of the electrostatic free energy of binding as a function of salt concentration and protein charge. Considering the electrostatic interactions to depend primarily on charge/charge, charge/dipole and dipole/dipole terms, we apply a generalization of a DLVOlike model augmented with dipolar terms as discussed previously (Bratko et al., 2002). The model estimates the orientation-averaged potential of mean force between charged polar particles as a sum of independent charge/charge $(q q)$, charge/dipole $(q \mu)$ and dipole/dipole $(\mu \mu)$ 
contributions (Phillies, 1974; Coen et al., 1995), corrected by a perturbation term (Bratko et al., 2002) that accounts for the coupling between interdependent charge/dipole and dipole/dipole terms. To date, this theoretical description has been applied only to a pair of identical proteins (Bratko et al., 2002) where the perturbation term is always repulsive. Below, we present a generalization to an asymmetric case, with interacting particles $i$ and $j$ carrying different charges, $q_{\mathrm{l}}, q_{\mathrm{j}}$, and dipole moments, $\mu_{\mathrm{l}}, \mu_{\mathrm{j}}$. This generalization leads to a new expression for the coupling perturbation term (Eq. 12) that may bring a qualitatively different effect of coupling between the three dipole-related contributions. We begin our analysis by expressing the electrostatic potential of mean force $w_{i j}\left(r_{i j}\right)$ as the sum of a direct charge/charge term, orientation averaged charge/dipole term (Miklavcic, 1998) and dipole/dipole interaction term (Phillies, 1974; Coen et al., 1995) and perturbation $w_{p}\left(r_{i j}\right)$ (Bratko et al., 2002) :

$w_{i j}\left(r_{i j}\right)=u_{q_{i} q_{j}}\left(r_{i j}\right)+w_{q_{i} \mu_{j}}\left(r_{i j}\right)+w_{\mu_{i} q_{j}}\left(r_{i j}\right)+w_{\mu_{i} \mu_{j}}\left(r_{i j}\right)+w_{p}\left(r_{i j}\right)$

where

$u_{q_{i} q_{j}}\left(r_{i j}\right)=\frac{q_{i} q_{j}}{4 \pi \varepsilon \varepsilon_{o} r_{i j}} S_{o}\left(r_{i j}, \kappa\right)$

$w_{q_{i} \mu_{j}}\left(r_{i j}\right)=-k_{B} T \ln \left[\frac{1}{\alpha_{i j}^{1}\left(r_{i j}\right)} \sinh \alpha_{i j}^{1}\left(r_{i j}\right)\right], \quad \alpha_{i j}^{1}\left(r_{i j}\right)=\frac{q_{i} \mu_{j}}{4 \pi \varepsilon \varepsilon_{0} r_{i j}^{2} k_{B} T} S_{1}\left(r_{i j}, \kappa\right)$

$w_{\mu_{i} q_{j}}\left(r_{i j}\right)=-k_{B} T \ln \left[\frac{1}{\alpha_{j i}^{1}\left(r_{i j}\right)} \sinh \alpha_{j i}^{1}\left(r_{i j}\right)\right], \quad \alpha_{j i}^{1}\left(r_{i j}\right)=\frac{q_{j} \mu_{i}}{4 \pi \varepsilon \varepsilon_{0} r_{i j}^{2} k_{B} T} S_{1}\left(r_{i j}, \kappa\right)$

and

$$
\begin{aligned}
& w_{\mu_{i} \mu_{j}}\left(r_{i j}\right)=-k_{B} T\left[\frac{2 \alpha_{i j}^{2}\left(r_{i j}\right)^{2}+\alpha_{i j}^{3}\left(r_{i j}\right)^{2}}{9}\right], \\
& \alpha_{i j}^{2}\left(r_{i j}\right)=\frac{\mu_{i} \mu_{j}}{4 \pi \varepsilon \varepsilon_{0} r_{i j}^{3} k_{B} T} S_{2}\left(r_{i j}, \kappa\right), \quad \alpha_{i j}^{3}\left(r_{i j}\right)=\frac{\mu_{i} \mu_{j}}{4 \pi \varepsilon \varepsilon_{0} r_{i j}^{3} k_{B} T} S_{3}\left(r_{i j}, \kappa\right) .
\end{aligned}
$$


In these equations, $r_{i j}$ is the center-to-center distance between the two particles, $\varepsilon$ is the dielectric constant of the solvent, $\varepsilon_{o}$ is the permittivity of vacuum; $k_{B} T$ is the thermal energy, and $S_{k}\left(r_{i j}, \kappa\right)$ stands for screening functions (Phillies, 1974) for charge/charge $(k=0)$, charge/dipole $(k$ $=1)$, and dipole/dipole $(k=2,3)$ interactions given by Eqs. 4-7 of Bratko et al. (2002). In addition to the distance, $r_{i j}$, these functions depend on the Debye screening parameter $\kappa \sim 0.33 \sqrt{I}$, where $I=(1 / 2) \Sigma c_{i} z_{i}^{2}$ is the ionic strength of the solution (Friedman, 1985), and on the dielectric constant of the solute interior, $\varepsilon_{s}$. The coupling correction $w_{p}\left(r_{i j}\right)$ is estimated according to the discrete orientation model introduced in Bratko et al. (2002). The model considers only six principal orientations of the dipoles, giving a partition function with thirty-six angle-dependent contributions. For two identical particles, the symmetry of the model leads to a degeneracy that reduces the number of independent energy levels to eight. In the more general scenario $\left(\alpha_{i j}^{1}\right.$ $\left.\neq \alpha_{j i}^{1}\right)$ considered in this work, the number of different energy levels is increased to eleven. Following the analysis of Bratko et al. (2002), we obtain $w_{d}\left(r_{i j}\right)$, the discrete orientation approximation to $w_{i j}\left(r_{i j}\right)$ :

$$
\begin{aligned}
w_{d}\left(r_{i j}\right)= & u_{q_{i} q_{j}}\left(r_{i j}\right)-k_{B} T \ln \left[8+4 e^{\alpha_{i j}^{1}}+4 e^{-\alpha_{i j}^{1}}+4 e^{\alpha_{j i}^{1}}+4 e^{-\alpha_{j i}^{1}}+4 e^{\alpha_{i j}^{3}}+4 e^{-\alpha_{i j}^{3}}\right. \\
& \left.+e^{-2 \alpha_{i j}^{2}-\left(\alpha_{i j}^{1}-\alpha_{j i}^{1}\right)}+e^{2 \alpha_{i j}^{2}-\left(\alpha_{i j}^{1}+\alpha_{j i}^{1}\right)}+e^{2 \alpha_{i j}^{2}+\left(\alpha_{i j}^{1}+\alpha_{j i}^{1}\right)}+e^{-2 \alpha_{i j}^{2}+\left(\alpha_{i j}^{1}-\alpha_{j i}^{1}\right)}\right] .
\end{aligned}
$$

We use Eq. 11 to obtain the coupling correction $w_{p}\left(r_{i j}\right)$ :

$$
\begin{aligned}
& w_{p}\left(r_{i j}\right)=w_{d}\left(r_{i j}, \alpha_{i j}^{1}, \alpha_{j i}^{1}, \alpha_{i j}^{2}, \alpha_{i j}^{3}\right)-w_{d}\left(r_{i j}, 0,0, \alpha_{i j}^{2}, \alpha_{i j}^{3}\right)-w_{d}\left(r_{i j}, \alpha_{i j}^{1}, \alpha_{j i}^{1}, 0,0\right)= \\
& -k_{B} T \ln \frac{18\left[4+4 \cosh \alpha_{i j}^{1}+4 \cosh \alpha_{j i}^{1}+4 \cosh \alpha_{i j}^{3}+e^{-2 \alpha_{i j}^{2}} \cosh \left(\alpha_{i j}^{1}-\alpha_{j i}^{1}\right)+e^{2 \alpha_{i j}^{2}} \cosh \left(\alpha_{i j}^{1}+\alpha_{j i}^{1}\right)\right]}{\left[8+4 \cosh \alpha_{i j}^{1}+4 \cosh \alpha_{j i}^{1}+\cosh \left(\alpha_{i j}^{1}-\alpha_{j i}^{1}\right)+\cosh \left(\alpha_{i j}^{1}+\alpha_{j i}^{1}\right)\right]\left[12+4 \cosh \alpha_{i j}^{3}+2 \cosh \left(2 \alpha_{i j}^{2}\right)\right]} .(12)
\end{aligned}
$$


For a symmetric case, $\alpha_{i j}^{1}=\alpha_{j i}^{1}$, Eq. 12 simplifies to Eq. 21 of Bratko et al. (2002) for identical interacting particles. In the latter case, when both proteins carry charges of the same sign, the coupling correction is repulsive because charge/dipole and dipole/dipole interactions favor opposite orientations. In a more general case, when the two charges may be of opposite signs, e.g., for the DnaK/peptide binding considered here, the two types of interaction can enhance each other, leading to stronger binding than that predicted by the conventional additivity approximation (Coen et al., 1995). In the following section, we use complete Eq. 7, including the coupling term given by Eq. 12, to interpret the measured electrostatic binding free energies.

\section{Parameters for the Potential of Mean Force}

The charges and dipole moments of the protein and peptide were taken from the online Weizmann Institute database (http://bioinformatics.weizmann.ac.11/dipol; Felder et al., 1997; Botti et al., 1998), based on protein crystal structures from the Protein Data Bank (PDB) and using the Parse (Sitkoff et al., 1994) set of partial atomic charges at pH 7. In our calculations, we use the crystal structure data for the DnaK substrate-binding domain complex with peptide NRLLLTG from PDB (1DKX.PDB; Zhu et al, 1996). The DnaK substrate-binding domain carries a total charge of $-10 e_{o}$, where $e_{o}$ is the proton charge, and a dipole moment of 542 Debye. The peptide NRLLLTG has a total charge of $+1 e_{o}$ and a dipole moment of 140 Debye. In our calculations, we determine the electrostatic free energy of binding as the electrostatic contribution to the potential of mean force between DnaK and peptide molecules at contact, that is, when $r_{12}=\sigma_{12}=\left(\sigma_{1}+\sigma_{2}\right) / 2$. In view of the deviation of molecular shapes for both DnaK and peptide from spherical geometry, molecular diameters, $\sigma_{l}$ and $\sigma_{2}$, were approximated by the diameters of equivalent spheres, i.e., spheres whose volumes are equal to those of the DnaK and 
the peptide molecules, respectively. This procedure leads to the effective sizes $\sigma_{l} \sim 1.4 \mathrm{~nm}$ for the peptide and $\sigma_{2} \sim 4 \mathrm{~nm}$ for the DnaK substrate binding domain. The DnaK ATPase domain is not involved in substrate binding directly and bears little effect on the electrostatic interaction, but may increase the overall excluded volume effect. Including the volume of ATPase domain increases the effective DnaK diameter to $\sim 5 \mathrm{~nm}$. As shown below, the latter value leads to better agreement between calculated and measured electrostatic free energies of DnaK/NRLLLTG binding. The increase in the effective contact distance may also partly be attributed to the presence of the dye (fluorescein) attached to the peptide. Analogous to earlier studies (Montgomery et al., 1999), our calculations presume no direct dye effect on peptide binding. Finally, a uniform dielectrict constant $\varepsilon_{p}=4$ was assigned to the interior of interacting solutes.

\section{Results and discussion:}

\section{Dissociation Constants and Determination of the Electrostatic Free Energy of Binding}

The dissociation constants for peptide f-NRLLLTG or peptide f-NALLLTG and DnaK were measured by fluorescence anisotropy in $20 \mathrm{mM}$ phosphate buffer with $\mathrm{NaCl}$ concentration at $0,0.1$, 0.5 and 1.0 M with results shown in Fig. 2. The interaction between peptide f-NRLLLTG and DnaK showed a strong dependence on ionic strength. In $20 \mathrm{mM}$ phosphate buffer, dissociation constants increase from $0.2 \pm 0.02 \mu \mathrm{M}$ in the absence of sodium chloride to $1.12 \pm 0.05 \mu \mathrm{M}$ in $1 \mathrm{M}$ sodium chloride due to electrostatic screening of Coulombic attraction.

By substituting Arg with Ala in peptide f-NALLLTG, we eliminate the positive charge. The resulting dissociation constant for the f-NALLLTG/DnaK complex showed only a very weak dependence on the ionic strength because the primary interaction is hydrophobic, with 
binding strength very close to that of f-NRLLLTG with DnaK in the high-ionic-strength solution. This result confirmed that the electrostatic interaction between f-NRLLLTG and DnaK was completely screened in $1 \mathrm{M}$ sodium chloride. Measurements for NALLLTG and DnaK also indicate that hydrophobic interaction is not appreciably affected by the presence of salt.

Like f-NRLLLTG, the peptide f-NRAAATG carries a single positive charge and is expected to feature a similarly strong electrostatic interaction with DnaK. Substituting leucine residues by alanine, however, considerably reduces the hydrophobic character of the peptide. Fluorescence anisotropy data for f-NRAAATG revealed essentially no binding to DnaK (data not shown), indicating the essential role hydrophobic interactions play in DnaK/peptide association.

From the dissociation constants for f-NRLLLTG and DnaK, the electrostatic binding free energies were determined to be $-1.0 \mathrm{kcal} / \mathrm{mol}$ using the relation: $\Delta G_{\text {ele }}=R T \ln \left(K_{d 2} / K_{d 1}\right)$, where $K_{d 2}$ and $K_{d l}$ are the dissociation constants at high and low ionic strength, respectively. The nonelectrostatic contribution (mainly hydrophobic interactions) was calculated from the dissociation constant at high ionic strength; it is $-8 \mathrm{kcal} / \mathrm{mol}$. In a previous study of the interaction between DnaK and acrylodan-labeled peptide NRLLLTG, the dissociation constant $K_{d}$ was about $0.2 \mu \mathrm{M}$ in the assay buffer of $25 \mathrm{mM}$ Hepes/ $\mathrm{NaOH}, 100 \mathrm{mM} \mathrm{KCl}, \mathrm{pH} \sim 7.0$ (Pierpaoli et al., 1998). From our data, $K_{d}$ for f-NRLLLTG with DnaK is about $0.2 \pm 0.02 \mu \mathrm{M}$ in $20 \mathrm{mM}$ phosphate buffer, and about $0.7 \pm 0.05 \mu \mathrm{M}$ in $20 \mathrm{mM}$ phosphate buffer with $100 \mathrm{mM} \mathrm{NaCl}$. Neither acrylodan nor fluorescein has been found to have any specific DnaK binding affinity (Pierpaoli et al., 1998; Montgomery et al., 1999). It is not clear if different types of salt ions present in the solution might cause the above discrepancy in dissociation constants. 


\section{Model Calculations}

Fig. 3 shows the ionic-strength dependence of the electrostatic free energy of DnaK/NRLLLTG binding predicted by Eqs. 7-12 using the model parameters described above along with the effective DnaK/peptide contact distance $\sigma_{12} \sim 3.2 \mathrm{~nm}\left(\sigma_{2} \sim 5 \mathrm{~nm}\right)$ (solid curve). For comparison, Fig. 3 includes a result based on the pairwise additivity approximation obtained by setting the coupling correction $w_{p}\left(\sigma_{12}\right)=0$ (dashed curve). The shapes of both curves are consistent with the experimental electrostatic free energy (shown in symbols) obtained from dissociation-constant measurements. The two curves in Fig. 3 are almost indistinguishable at high screening, characterized by a weak coupling between the distinct contributions, but differ by about $40 \%$ at low ionic strength $(I \sim 0.04 \mathrm{M})$. This highlights the importance of the coupling term for accurate estimates of binding strengths when electrostatic interactions are not significantly reduced by screening. The sign of the coupling correction is negative, in contrast to previously studied cases where both particles are either negatively or positively charged (Bratko et al., 2002); for such cases, the correction is always repulsive. In view of this distinction, an accurate account of the coupling term is crucial for predicting the outcome of a $\mathrm{pH}$ titration that brings about charge reversal on either of the interacting species.

Equations 8-10 predict a strong dependence of binding electrostatics on the contact distance of interacting molecules. The effect of the effective hard-sphere separation for a bound pair, $\sigma_{12}$, is shown in the inset of Fig. 3 where we compare the ionic-strength dependence of the electrostatic free energy of binding for DnaK/NRLLLTG using the effective size of the entire DnaK molecule, or the smaller value corresponding to the substrate binding domain. In the former case (solid curve), $\sigma_{12} \sim 3.2 \mathrm{~nm}$ and in the latter $\sigma_{12} \sim 2.7 \mathrm{~nm}$ (dash-doted curve). Comparison with the experimental dependence presented in Fig. 3 shows excellent agreement 
between the experiment and theory when the higher $\sigma_{12}$ is used. Both curves in the inset of Fig. 3 reproduce qualitatively the ionic-strength dependence observed experimentally. As different contributions to the total electrostatics (charge/charge, charge/dipole and dipole/dipole) feature different ionic-strength dependencies (Phillies, 1974; Bratko et al. 2002), it appears that our approximate model captures the essential contributions in correct proportions. Uncertainties associated with the choice of the appropriate value for the effective contact separation, $\sigma_{12}$, can be resolved by calibration, adjusting $\sigma_{12}$ to reproduce the measured interaction strength at low salt concentration. Ignoring presumably weak structural dependence on ionic strength or solution

$\mathrm{pH}$, the same apparent sizes can then be applied for calculations at different solution conditions or with another peptide of similar size. Applications of considerably more detailed models based on complete molecular geometries do not remove the need for similar calibrations (Gilson et al., 1988). Success of the simplified approach that considers only the leading electrostatic contributions, as illustrated in the above example, along with the advantage of computational simplicity, suggests that our generalization of the electrostatic model of Bratko et al. (2002) provides a useful tool toward obtaining good estimates of the strength of electrostatic binding between different protein/substrate pairs.

\section{Conclusion}

Dnak recognizes peptides with internal hydrophobic residues and positively charged terminal residues. The peptide NRLLLTG has a strong binding affinity for DnaK; the complex formed with the DnaK substrate-binding domain has been crystallized (Zhu et al., 1996). The positively charged residues of the peptide interact with the negatively charged cluster in the 
peptide-binding site of DnaK (Zhu et al., 1996). To shed light on the interactions between molecular chaperones and polypeptides, and especially to delineate the electrostatic contribution to their association, we determined the ionic strength dependence of dissociation constants for DnaK complexes with selected oligopeptides NRLLLTG, NRAAATG, and NALLLTG. The nonelectrostatic contribution to the free energies of binding between DnaK and ionic peptide NRLLLTG (determined from a comparison with electrostatically screened systems) agree with the corresponding value for the nonionic analogue of NRLLLTG, NALLLTG. While hydrophobic interaction dominates the chaperone/peptide interaction, the electrostatic contribution of $-1.1 \mathrm{kcal} / \mathrm{mol}$ (close to $-2 R T$ ), is sufficient to affect significantly the strength of association. Because we can easily modify interprotein electrostatics, e.g. by ionic strength or $\mathrm{pH}$ variation, electrostatic interactions represent a promising tool to alter chaperone/peptide binding. To facilitate control of interprotein electrostatics, we have developed an approximate analytic model for the prediction of the electrostatic contribution to the potential of mean force for a pair of dissimilar dipolar particles. For oppositely charged species, the model predicts a significant enhancement of interparticle attraction due to the cooperativity between charge/dipole and dipole/dipole interactions. The calculated electrostatic free energy of binding shows reasonable agreement with that obtained from fluorescence-anisotropy measurements 


\section{Acknowledgment}

The authors are grateful to the National Science Foundation under Grant No. BES0118208 and to the Office of Basic Energy Sciences of the U.S. Department of Energy for financial support, to Dr. Michael Moore for synthesis of peptides, and to Prof. François Baneyx for providing plasmid $\mathrm{pDnaK}$. 


\section{References}

Asthagiri, D., B. L. Neal, and A. M. Lenhoff. 1999. Calculation of short-range interactions between proteins. Biophys. Chem. 78:219-231

Botti, S. A., C. E. Felder, J. L. Sussman, and I. Silman. 1998. Electrotactins: a class of proteins with conserved electrostatic and structural motifs. Protein Eng. 11:415-420

Bratko, D., A. Striolo, J. Z. Wu, H. W. Blanch, and J. M. Prausnitz. 2002. Orientation-averaged pair potentials between dipolar proteins or colloids. J. Phys. Chem. B. 106:2714-2720

Cegielska, A., and C. Georgopoulos. 1989. Functional domains of the Escherichia coli dnaK heat shock protein as revealed by mutational analysis. J. Biol. Chem. 264:21122-21130

Chaykin, S. 1966. Biochemistry laboratory techniques. Wiley, New York

Coen, C. J., H. W. Blanch, and J. M. Prausnitz. 1995. Salting out of aqueous proteins: phase equilibria and intermolecular potentials. AICHE J. 41:996-1004

Coen, C. J., J. Newman, H. W. Blanch and J. M. Prausnitz. 1996. Electrostatic protein-protein interactions: Comparison of point-dipole and finite-length dipole potentials of mean force. $J$. Colloid Interface Sci. 177:276-279

Einstein, A. 1906. Zur theorie der Brownschen bewegung. Annalen der Physik. 19:371-381

Farnum, M. A., and C. F. Zukoski. 1999. Effect of glycerol on the interactions and solubility of bovine pancreatic trypsin inhibitor. Biophys. J. 76:2716-2726

Felder, C. A., S. A. Botti, S. Lifson, I. Silman, and J. L. Sussman. 1997. External and internal electrostatic potentials of cholinesterase models. J. Mol. Graph. Model. 15:318-327

Fourie, A. M., J. F. Sambrook, and M. H. Gething. 1994. Common and divergent peptide binding specificities of hsp70 molecular chaperones. J. Biol. Chem. 269:30470-30478 
Friedman, H. L. 1985. A course in statistical mechanics. Prentice Hall, Englewood Cliffs Gething, M., and J. Sambrook. 1992. Protein folding in the cell. Nature. 355:33-45

Gilson, M. K., K. A. Sharp, and B. H. Honig. 1988. Calculating the electrostatic potential of molecules in solution - method and error assessment. J. Comput. Chem. 9:327-335

Gragerov, A., L. Zeng, X. Zhao, W. Burkholder, and M. E. Gottesman. 1994. Specificity of DnaK-peptide binding. J. Mol. Biol. 235:848-854

Grant, M. L. 2001. Nonuniform charge effects in protein-protein interactions. J. Phys. Chem. B. $105: 2858-2863$

Hartl, F. U. 1996. Molecular chaperones in cellular protein folding. Nature. 381:571-580

Kamath-Loeb, A. S., C. Z. Lu, W. Suh, M. A. Lonetto, and C. A. Gross. 1995. Analysis of three DnaK mutant proteins suggests that progression through the ATPase cycle requires conformational changes. J. Biol. Chem. 270:30051-30059

Kasper, P., P. Christen, and H. Gehring. 2000. Empirical calculation of the relative free energies of peptide binding to the molecular chaperone DnaK. Proteins: Struct. Funct. Genet. 40:185-192 Lakowicz, J. R. 1983. Principles of fluorescence spectroscopy. Plenum Press, New York Langer, T., C. Lu, H. Echols, J. Flanagan, M. K. Hayer, and F. U. Hartl. 1992. Successive action of DnaK, DnaJ and GroEL along the pathway of chaperone-meditated protein folding. Nature. $356: 683-689$

Lundblad, J. R., M. Laurence, R. H. Goodman. 1996. Fluorescence polarization analysis of protein-DNA and protein-protein interactions. Mol. Endocrinol. 10:607-612

Mayer, M. P., S. Rüdiger, and B. Bukau. 2000. Molecular basis for interactions of the DnaK chaperone with substrates. Biol. Chem. 381:877-885 
McClurg, R. B., and C. F. Zukoski. 1998. The electrostatic interaction of rigid, globular proteins with arbitrary charge distributions. J. Colloid Interface Sci. 208:529-542

Miklavcic, S. J. 1997. Effective dipole potentials after angle averaging. Phys. Rev. E. 56:11421147

Montgomery, D. L., R. I. Morimoto, and L. M. Gierasch. 1999. Mutations in the substrate binding domain of the Escherichia coli $70 \mathrm{kDa}$ molecular chaperone, DnaK, which alter substrate affinity or interdomain coupling. J. Mol. Chem. 286:915-932

Neal, B. L., D. Asthagiri, O. D. Velev, A. M. Lenhoff, and E. W. Kaler. 1999. Why is the osmotic second virial coefficient related to protein crystallization? J. Crystal Growth. 196:377387

Nicholls, A., and B. Honig. 1991. A rapid finite-difference algorithm, utilizing successive overrelaxation to solve the Poisson-Boltzmann equation. J. Comput. Chem. 12:435-445

Nicholls, A., K. A. Sharp, and B. Honig. 1991. Protein folding and association - insights from the interfacial and thermodynamic properties of hydrocarbons. Proteins: Struct. Funct. Genet. $11: 281-296$

Novotny, J., R. E. Bruccoleri, M. Davis, and K. A. Sharp. 1997. Empirical free energy calculations: a blind test and further improvements to the method. J. Mol. Biol. 268:401-411 Phillies, G. D. J. 1974. Excess chemical potential of dilute solutions of spherical polyelectrolytes. J. Chem. Phys. 60:2721-2731

Pierpaoli, E. V., E. Sandmeier, A. Baici, H. Schönfeld, S. Gisler, and P. Christen. 1997. The power stroke of the DnaK/DnaJ/GrpE molecular chaperone system. J. Mol. Biol. 269:757-768 Pierpaoli, E. V., S. M. Gisler, and P. Christen. 1998. Sequence-specific rates of interaction of target peptides with the molecular chaperones DnaK and DnaJ. Biochemistry. 37: 16741-16748 
Rüdiger, S., A. Buchberger, and B. Bukau. 1997a. Interaction of Hsp70 chaperones with substrates. Nat. Struct. Biol. 4:342-349

Rüdiger, S., L. Germeroth, J. Schneider-Mergener, and B. Bukau. 1997b. Substrate specificity of the DnaK chaperone determined by screening cellulose-bound peptide libraries. EMBO J. $16: 1501-1507$

Sader, J. E., and A. M. Lenhoff. 1998. Electrical double-layer interaction between heterogeneously charged colloidal particles: A superposition formulation. J. Colloid Interface Sci. 201:233-243

Sheinerman, F. B., R. Norel, and B. Honig. 2000. Electrostatic aspects of protein-protein interactions. Curr. Opin. Struct. Biol. 10:153-159

Sitkoff, D., K. A. Sharp, B. Honig. 1994. Accurate calculation of hydration free energies using macroscopic solvent models. J. Phys. Chem. 98:1978-1988

Smith, P. K., R. I. Krohn, G. T. Hermanson, A. K. Malia, F. H. Gartner, M. D. Provenzano, E. K. Fujimoto, N. M. Goeke, B. J. Olson, and D. C. Klenk. 1985. Measurement of protein using bicinchoninic acid. Anal. Biochem. 150:76-85

Tardieu, A., A. L. Verge, M. Malfois, F. Bonneté, S. Finet, M. Riès-Kautt, and L. Belloni. 1999. Proteins in solution: from X-ray scattering intensities to interaction potentials. J. Crystal Growth. 196:193-203

Wang, H., A. V. Kurochkin, Y. Pang, W. Hu, G. C. Flynn, and E. R. P. Zuiderweg. 1998. NMR solution structure of the $21 \mathrm{kDa}$ chaperone protein DnaK substrate binding domain: a preview of chaperone-protein interaction. Biochemistry. 37:7929-7940

Weber, G. 1953. Rotational Brownian motion and polarization of the fluorescence of solutions. Adv. Prot. Chem. 8:415-459 
Zhu, X., X. Zhao, W. F. Burkholder, A. Gragerov, C. M. Ogata, M. E. Gottesman, and W. A. Hendrickson. 1996. Structural analysis of substrate binding by the molecular chaperone DnaK. Science. 272:1606-1614 


\section{Figure Captions:}

Figure1. Fluorescence-anisotropy measurement. The anisotropy, $A$, is measured as a function of DnaK concentration in $20 \mathrm{mM}$ phosphate buffer at $\mathrm{pH} 7$ and various $\mathrm{NaCl}$ concentrations. The fluorescence-labeled peptide concentrations was $2 \mathrm{nM}$; (a) f-NRLLLTG, (b) f-NALLLTG.

Figure 2. Dissociation constants for f-NRLLLTG and f-NALLLTG/DnaK complexes as a function of $\mathrm{NaCl}$ concentration in $20 \mathrm{mM}$ phosphate buffer at $\mathrm{pH} 7$.

Figure 3. Comparison of ionic-strength dependence of the electrostatic free energy, $\Delta G_{\text {ele }}$, calculated from the additivity approximation (dashed line); from the present model with chargedipole coupling (solid line); and from dissociation-constant measurements (symbols). In these calculations, the DnaK has a diameter of $5 \mathrm{~nm}$, and the contact distance of DnaK/peptide is 3.2 nm. Inset: Electrostatic free energy of binding from the approximate analytic model for DnaK with diameter $5 \mathrm{~nm}$ (solid line) and $4 \mathrm{~nm}$ (dash-dot line) as a function of ionic strength $I$. 


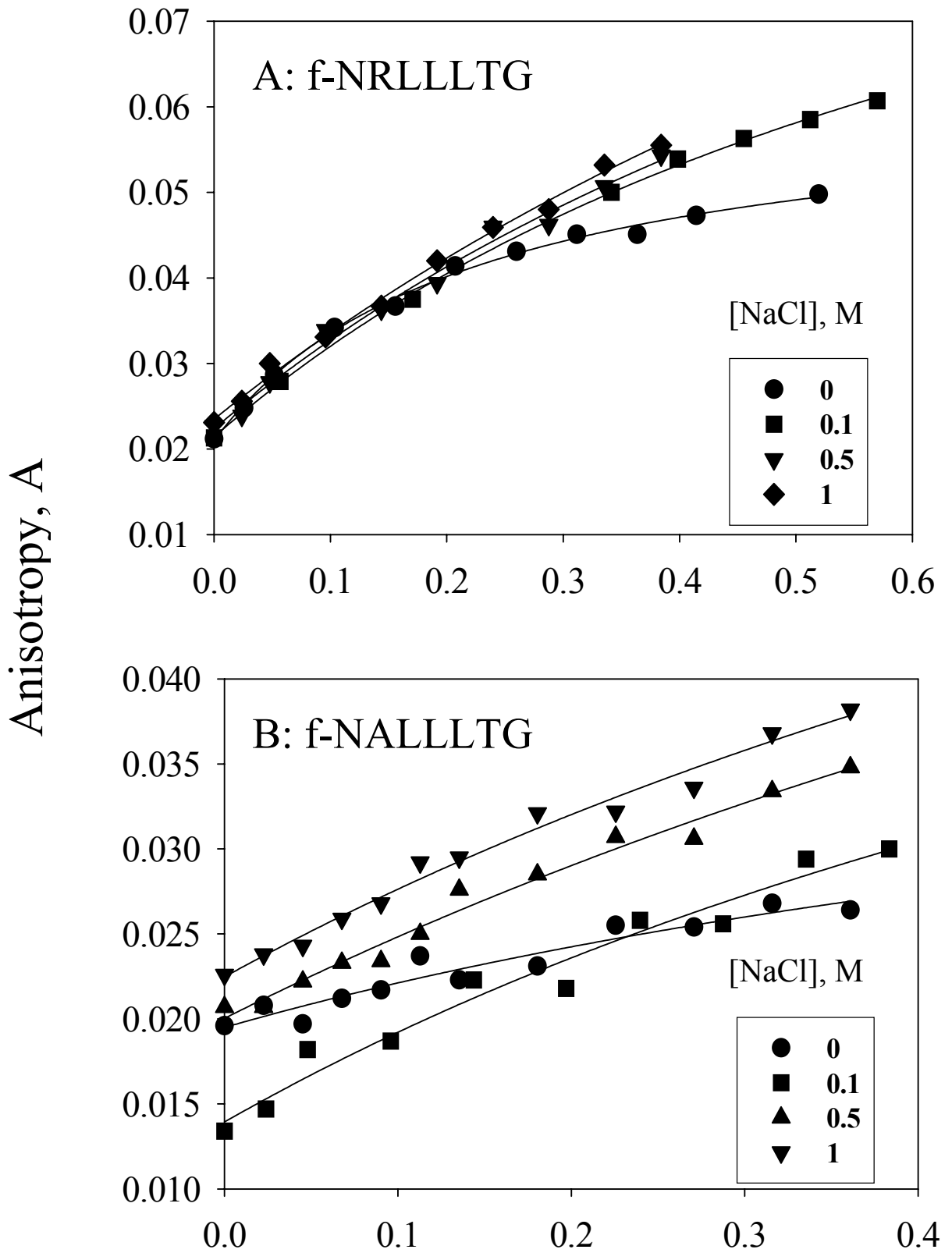

Concentration of DnaK $[\mu \mathrm{M}]$

Liu et al., figure 1 


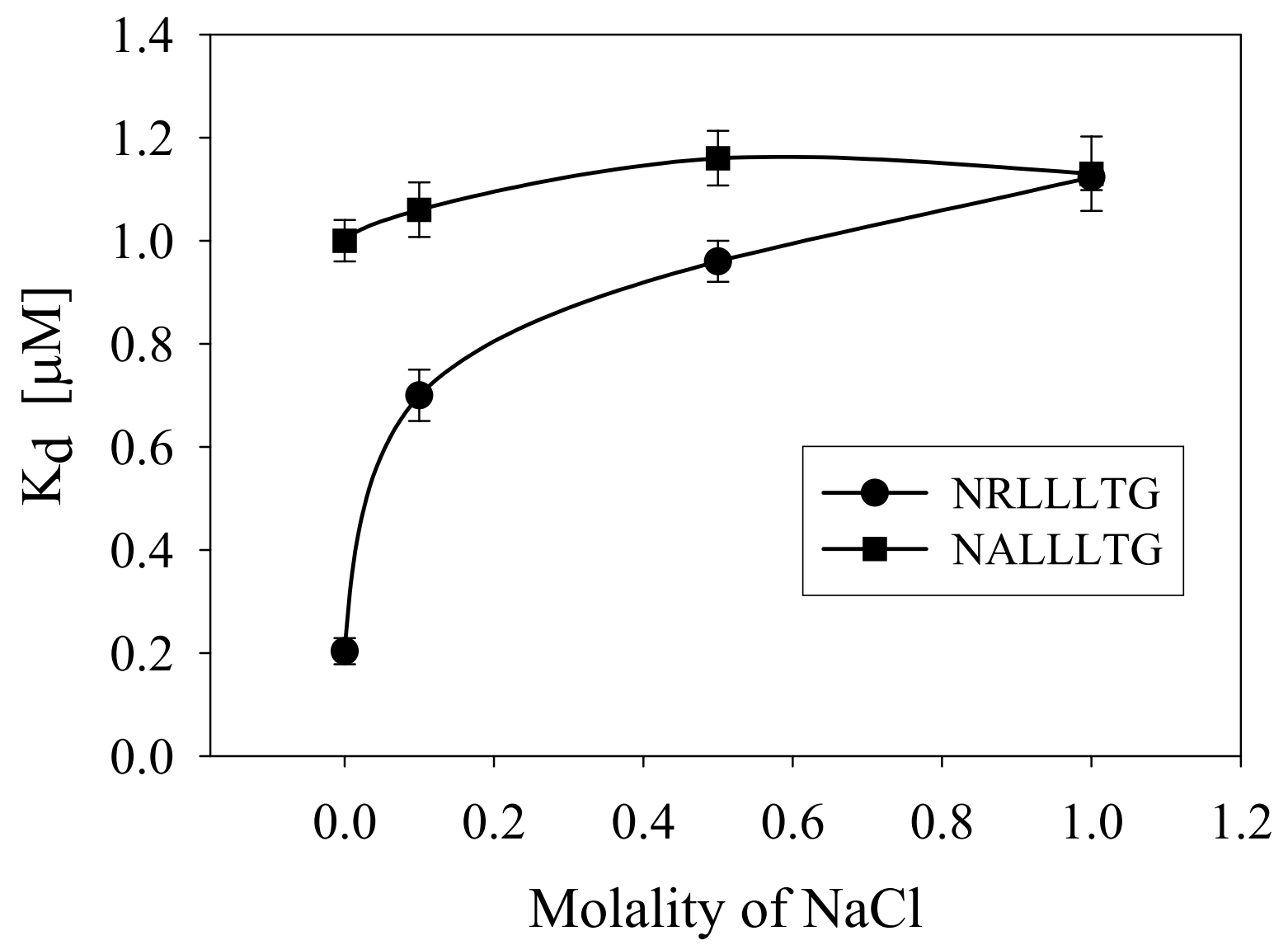

Liu et al., figure 2 
Liu et al., figure 3 